\title{
Ghrelin: a step forward in the understanding of somatotroph cell function and growth regulation
}

\author{
C Dieguez $^{1}$ and F F Casanueva ${ }^{2}$ \\ Departments of ${ }^{1}$ Physiology and ${ }^{2}$ Medicine, Faculty of Medicine, Santiago de Compostela University, Santiago de Compostela, Spain \\ (Correspondence should be addressed to C Dieguez, Faculty of Medicine, University of Santiago, P.O. Box 563, 15700 Santiago de Compostela, Spain; \\ Email: fscadigo@usc.es)
}

\section{Historical background}

In 1977 CY Bowers and coworkers developed a series of small peptides which stimulated in vitro the release of growth hormone (GH) from pituitary cells, although their potency was rather weak (1). Further studies based on conformational energy calculations in conjunction with peptide chemistry modifications and biological activity testing, led in 1984 to the development of several more potent peptides, including growth hormone-releasing peptide-6 (GHRP-6) (2). This hexapeptide was shown to be a potent releaser of GH both in vivo and in vitro in all tested animal species. Furthermore, this compound was shown to be active when administered by different routes such as i.v., i.p., i.m. and even by the oral route (3-4), thus becoming the standard reference compound for $\mathrm{GH}$ secretagogues (GHS). Unfortunately, this development was largely unnoticed by the scientific community at the time it was published, the reason being that in 1982, growth hormone-releasing hormone (GHRH) was characterized, and the work of most basic and clinical research centres was focused on assessing the role of GHRH in the regulation of somatotroph cell function, its mechanism of action as well as its diagnostic and therapeutic implications. In fact, it became dogma that GH secretion by the anterior pituitary gland was the net result of the antagonistic actions of two hypothalamic hormones - GHRH and somatostatin - which reach their receptors on the somatotroph cells via portal vessels. Thus, GHRH and somatostatin will be the final integrative pathway for the pertinent information reaching the hypothalamus. Different neurotransmitters and a vast array of metabolic and hormonal signals originated in peripheral tissues would influence GH secretion by regulating hypothalamic GHRH and somatostatin release and/or by modulating their effect directly on the somatotrophs. Nonetheless, it soon became apparent that the mechanism of action of GHRP-6 on GH secretion could not easily be explained according to the above mentioned model of $\mathrm{GH}$ secretion, and that GHRP-6 was not a surrogate of GHRH (3-6). The initial comparative analysis of the effects of GHRH and GHRP- 6 on GH secretion suggested that their mechanism of action was both different and complementary. Thus, while GHRH is a much more potent releaser of GH in vitro than GHRP-6, the opposite happens in vivo. Evidence for different mechanisms of action also derived from studies using maximally or sub maximally effective doses of GHRH and GHRP-6, which showed that the GH secretory responses in vivo were potentiated rather than additive. Besides, homologous but not heterologous desensitization could be shown after repetitive administration of these peptides. Finally, different mechanisms of action were confirmed through the identification of different receptors and intracellular signalling for GHRH and GHRP-6 (7). Nevertheless, there were still some uncertainties regarding the physiological relevance of this system due to the failure to isolate the endogenous ligand for this receptor(s) for GHRP-6.

Once it was clear that GHRP- 6 was a potent regulator of GH secretion acting through a different mechanism of action than GHRH, most pharmaceutical companies involved in the GH field and several independent groups developed and tested different GHRP- 6 analogues, also called GHS (Fig. 1), that were active orally, and had improved potency and bioavailibility. One of these developed compounds was MK-0677 that was used for the cloning of the only known GHS-receptor (7).

\section{The GHS-receptor}

A strategy of expression cloning was adopted by the group of R Smith, at that time at MSD Research Lab (USA), to isolate a cDNA encoding the GHS-receptor (7). In brief, this strategy was based on the findings that the signal transduction used by GHRP- 6 and similar compounds appeared to involve phospholipase $\mathrm{C}$ resulting in a rise in inositol trisphosphate and intracellular calcium. Xenopus oocytes were injected with swine poly $(\mathrm{A})^{+}$mRNA, as a source of GHS-receptor mRNA, supplemented with various G-alpha subunits mRNAs. Thereafter, they assessed $\mathrm{Ca}^{2+}$-activated $\mathrm{Cl}^{-}$ currents after being challenged with MK-0677. Using this approach they identified a single cDNA which encoded the GHS-receptor. This was found to be a classical G-protein coupled receptor, containing seven putative alpha-helical membrane spanning segments 


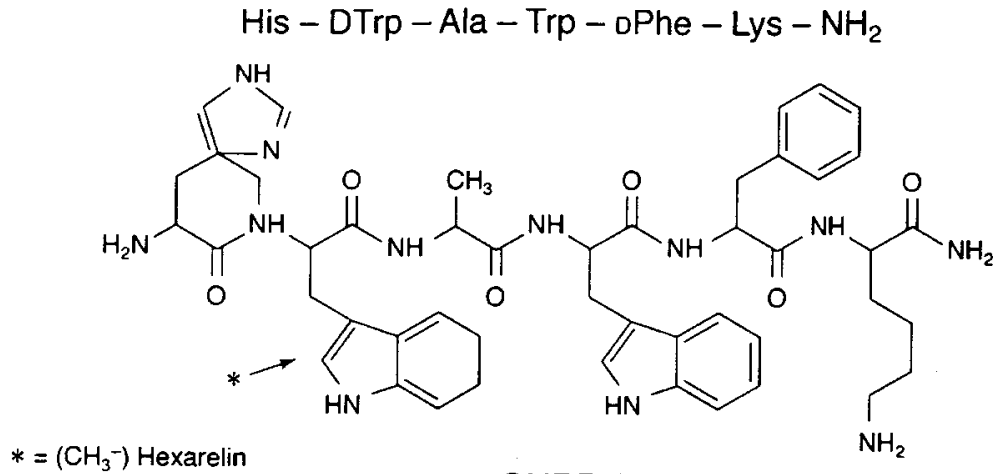

$*=\left(\mathrm{CH}_{3}^{-}\right)$Hexarelin

GHRP-6<smiles>Cc1ccc2ccccc2c1</smiles>

$4 b$<smiles>CC(C)(N)CC(=O)N[C@H]1CCc2ccccc2N(Cc2ccc(-c3ccccc3-c3nnn[nH]3)cc2)C1=O</smiles>

L-692,429 (MK-0751)<smiles>CN1CC2(CCN(C(=O)[C@H](COCc3ccccc3)NC(=O)C(C)(C)N)CC2)c2ccccc21</smiles>

L-163,191 (MK-0677)<smiles>CC(C)(N)C(=O)N[C@@H](Cc1c[nH]cn1)C(=O)N[C@@H](Cc1ccc2ccccc2c1)C(=O)N[C@@H](Cc1ccccc1)C(=O)N[C@@H](CCCN)C(N)=O</smiles>

NNC 26-0161

ipamorelin<smiles>CNC(=O)C(Cc1ccccc1)N(C)C(=O)[C@H](Cc1ccc2ccccc2c1)N(C)C(=O)/C=C/CC(C)(C)N</smiles>

NNC 26-0703

Figure 1 Structure of GHRP-6 and other synthetic growth hormone secretagogues all of which activate the cloned GHS-receptor. 
and three intracellular and extracellular loops. The full length human GHS-receptor encodes a 366 amino acid protein and is strongly conserved in evolution as assessed by the molecular analysis from swine, mouse, rat and human. In fact, the existence of this receptor can apparently be extended to PreCambrian times (400000 years) as amino acid sequences strongly related to the human GHS-receptor have been identified in teleost fish. Assessment of the tissue distribution of this receptor showed that it is mainly expressed in the anterior pituitary and the brain, in both hypothalamic and non hypothalamic regions. The wide expression of the receptor in the brain is in keeping with previous observations suggesting, for GHRP-6 and similar compounds, broader functions beyond the control of $\mathrm{GH}$ release (7).

\section{Isolation of ghrelin}

Despite intensive searches by different groups, the isolation of an endogenous ligand of the GHS-receptor remained elusive until recently. The cloning of the GHSreceptor was important because, in addition to the information in its own right, after expression in a cell line it could be used as an assay system to identify the unknown endogenous ligand. In this particular case the GHS-receptor was expressed in a $\mathrm{CHO}$ cell line to monitor changes in intracellular calcium concentrations that were induced by rat tissue extracts. Although this strategy was being used by several groups, the success of the group led by Kojima and Kangawa (8) is largely due to the fact that they tested extracts from different tissues such as brain, lung, heart, kidney and stomach. Despite the fact that most people assumed that the greatest concentrations would be in the hypothalamus, surprisingly, Kojima et al. found that the highest GHS-receptor activation was found in stomach extracts (8). The purified ligand was found to be a peptide of 28 amino acids, in which the serine 3 residue, and here is the second surprise, was n-octanoylated (Fig. 2). This peptide, now called GHRELIN (ghre is the Proto-Indo-European root of the word 'growth'), specifically releases GH both in vivo and in vitro, and the O-n-octanoylation appears to be essential for its activity, at least in terms of $\mathrm{GH}$ release. This was an intriguing finding since octanoylation had not been observed previously in peptide modification and opens up a new field in protein chemistry and in the development of biologically active peptides and peptidomimetic compounds.

Furthermore, the same group succeeded in isolating the gene coding for ghrelin (8). In both rats and humans, the gene encodes a prepro-ghrelin of 117 amino acids with an $82 \%$ homology, which indicates that ghrelins are highly conserved between species. Although detailed studies regarding the tissue distribution of this peptide are still needed, preliminary evidence indicates that the gene is expressed in both the stomach and the hypothalamic arcuate nucleus (8).

\section{Physiological and clinical implications}

The isolation of ghrelin is important for several reasons. It gives definitive proof of the existence of a GHS-GHSreceptor signalling system in the control of GH secretion. Basic questions such as whether the regulation a)

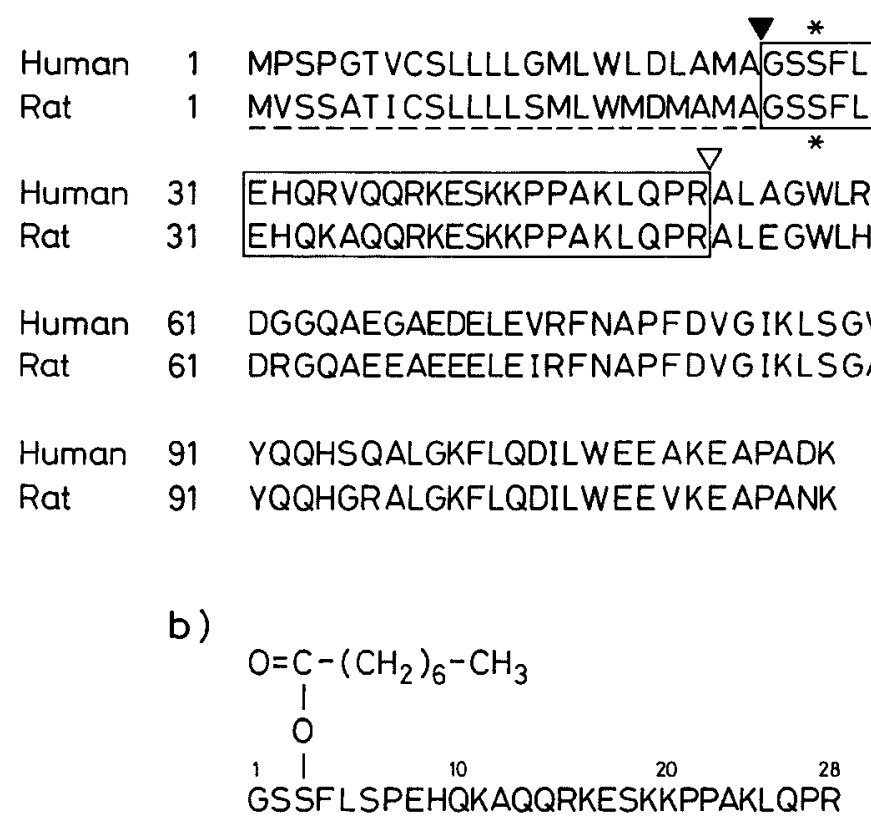

Figure 2 (a) Amino acid sequences of human and rat prepro-ghrelin. The open arrowhead indicates Ghrelin's last amino acid. The dotted line indicates a signal peptide. The filled arrowhead indicates a cleavage site of a signal peptide. Ghrelin sequences are boxed. Asterisks indicate n-octanoyl modification sites. (b) Structure of rat ghrelin (modified from reference 8 ). 
of $\mathrm{GH}$ secretion is due to stomach or arcuate nucleiderived ghrelin need to be addressed. By analogy with GHRH and somatostatin, also synthesized by peripheral tissues and present in the general circulation, one would expect that hypothalamic ghrelin would be responsible for the control of GH secretion, while other sources will influence other biological processes; however specific studies still need to be carried out. Questions regarding the mechanisms involved in the genesis of spontaneous $\mathrm{GH}$ secretion will have to be reviewed taking into account, in addition to GHRH and somatostatin, this new player (Fig. 3). In this regard, measurements of ghrelin in portal blood vessels and their relationship to $\mathrm{GH}$ pulses could clarify the relevance of $\mathrm{GHRH}$, somatostatin and ghrelin in $\mathrm{GH}$ pulsatility. In addition, passive immunization studies, as well as assessment of neuropeptide gene expression in normal rats as well as in some models of genetic and transgenic animals, will help to clarify the possible interaction between the three neuropeptides in terms of GH release. Also, measurements of ghrelin concentrations in plasma, for example after meals, must be recorded. Unravelling the mechanisms governing ghrelin-gene expression should help to clarify the involvement of ghrelin in some pathpophysiological settings as well as to answer some basic questions such as the sexual dimorphism of GH secretion. Further studies assessing the role of ghrelin in GH-gene transcription rate and somatotroph cell proliferation are also needed. It is already known that the GHSreceptor is expressed by different types of pituitary tumours. It is possible that, like GHRH, the ectopic production of ghrelin can lead to acromegaly. In fact, ghrelin can be measured in the peripheral circulation of normal subjects, and the existence of a circulating

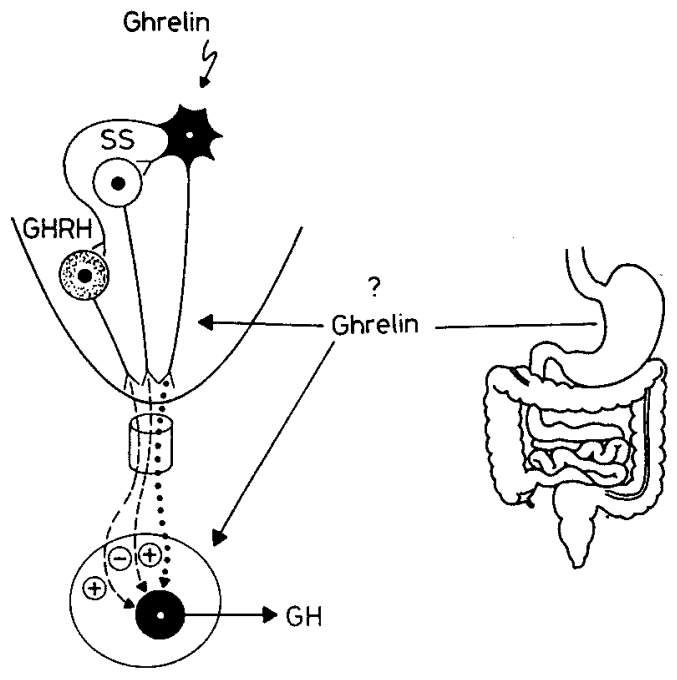

Figure $3 \mathrm{~A}$ new physiological model of the regulation of $\mathrm{GH}$ secretion involving ghrelin, GHRH and somatostatin (SS).
GH-stimulating factor other than GHRH in a patient with acromegaly has been shown previously (9). It is also easily imagined that deficiencies in ghrelin production and action might provide a logical explanation for some types of GH deficiency. Studies assessing the existence of mutation in the gene encoding ghrelin, as well as its knock-out in experimental animals, should answer this question.

In the same way that the cloning of the GHS-receptor was crucial for the isolation of ghrelin, this finding may well help in the characterization of new receptor subtypes. All the GHS compounds developed so far seem to exhibit a high binding affinity to the cloned GHS-receptor. However, there is strong evidence suggesting the existence of additional receptor subtypes which may exhibit different affinities for these compounds. Thus, in some tissues the specific binding of iodinated hexarelin can be displaced by peptidyl analogues of GHRP- 6 but not by MK-0677. Furthermore, although all peptidyl and non-peptidyl GHS are potent releasers of $\mathrm{GH}$, some of them are also able to stimulate the pituitary-adrenal axis leading to increased plasma adrenocorticotrophin (ACTH) and cortisol levels while others do not. Finally, the fact that knock-out mice of the cloned GHS-receptor exhibited a normal growth rate is compatible with the existence of redundant GHS-receptor subtypes that will still allow the biological effects of ghrelin. Therefore, it is likely that further subtypes of GHS are waiting to be cloned, and in this regard the use of their putative endogenous ligand, ghrelin, in the different assay systems may help to speed up the identification process. Furthermore, the comparison of the biological activity of ghrelin with some of the previously tested GHS compounds will clarify both the physiological relevance of some of the findings previously obtained as well as give a pharmacological profile indicative of the existence, or non-existence, of different receptor subtypes. In this regard, it will be interesting to compare the biological activity of ghrelin, in addition to GH secretion, in terms of the secretion of other hormones such as ACTH, cortisol and prolactin, in sleep control, food intake, gastrointestinal and cardiac function, since there is strong evidence that the biological activities of the ghrelin system may well be involved in the homeostasis of systems other than GH (10).

Data obtained so far assessing GHS compounds in the diagnostic setting have shown that the combined administration of GHRH and GHRP-6 (or similar compounds) appears to be the most potent stimulus of $\mathrm{GH}$ release in man, and it has been found to be a convenient, safe and reliable test for the diagnosis of adultonset GH deficiency (3). Also, there is a clear impression that there is a difference in $\mathrm{GH}$ responses to GHRH and GHS, in the sense that there is a large variability in the stimulatory action of GHRH contrasted with the greater reproducibility of GH responses to GHS. In different metabolic states, e.g. obesity and anorexia 
nervosa, the GH response after GHRH is more impaired than after GHS stimulation. On the other hand, in different neuroendocrine pathologies the $\mathrm{GH}$ response after GHS is more impaired than after GHRH. Thus, it will be interesting to see whether the marked differences in GH responses to GHRH and the synthetic GHS compounds in some clinical settings still remain when challenged with ghrelin.

Finally, the therapeutic possibilities of ghrelin, as well as new agonist and antagonist compounds, should be assessed. From the data available one would expect that, at least in terms of the $\mathrm{GH}$ axis, ghrelin will only be effective in subjects with a functional hypothalamic integrity, since the stimulatory effect of synthetic GHS compounds was strongly reduced in patients with pituitary stalk transection. However, the possibility of using ghrelin in aging patients as well as in patients with idiopathic GH deficiency, obesity, osteoporosis or cardiovascular diseases needs to be assessed (11). From an historical point of view, many will remember that the therapeutic potential of GHRH did not fulfil the earlier expectations. This does not imply that the same will happen with ghrelin, although it may.

In summary, the isolation of ghrelin can be considered a landmark in the GH field which opens up the possibility of gaining a greater insight into our understanding of the mechanisms involved in the regulation of $\mathrm{GH}$ secretion and somatic growth. The challenge now is to use the greater insight in knowledge that this discovery brings to improve the diagnosis and treatment of different disease states associated with altered GH secretion.

\section{Acknowledgements}

This work was supported by grants from Fondo de Investigaciòn Sanitaria, Spanish Ministry of Health and the Xunta de Galicia.

\section{References}

1 Bowers CY, Chang J, Momany F \& Folkers K. Effects of enkephalins and enkephalins analogs on release of pituitary hormones in vitro. In Molecular Endocrinology, pp 287-292. Ed. I MacIntyre. Amsterdam/North Holland: Elsevier, 1977.

2 Bowers CY, Momany F, Reynolds GA \& Hong A. On the in vitro and in vivo activity of a new synthetic hexapeptide that acts on the pituitary to specifically release growth hormone. Endocrinology 1984114 1537-1545.

3 Micic D, Casabiell X, Gualillo O, Pombo M, Dieguez C \& Casanueva FF. Growth hormone secretagogues: the clinical future. Hormone Research 199951 (Suppl 53) 29-33.

4 Ghigo E, Arvat E, Muccioli G \& Camanni F. Growth hormonereleasing peptides. European Journal of Endocrinology 1997136 445-460.

5 Oliver C, Dadoun F, Briard N, Guillaume V, Sauze N, Grino M et al. Mechanisms of action of growth hormone-releasing peptides and their analogues in vivo. In Growth Hormone Secretagogues, pp 105-114. Eds E Ghigo, M Boghen, FF Casanueva \& C Dieguez. Amsterdam: Elsevier, 1999.

6 Korbonits M \& Grossman AB. Growth hormone-releasing peptide and its analogues: novel stimuli to growth hormone release. Trends in Endocrinology and Metabolism 19956 43-49.

7 Smith RG, Van der Ploeg LHT, Howard AD, Freighner SD, Cheng K, Hickey GJ et al. Peptidomimetic regulation of growth hormone secretion. Endocrine Reviews 199718 621-645.

8 Kojima M, Hosoda H, Date Y, Nakazato M, Matsuo H \& Kangawa K. Ghrelin is a growth hormone-releasing acylated peptide from stomach. Nature 1999402 656-660.

9 Hulting AL, Wersall J \& Werner S. Evidence of a circulating growth hormone stimulating factor other than growth hormone releasing hormone in a patient with pituitary tumour and acromegaly. Acta Endocrinologica 1985109 289-294.

10 Nass RM \& Thorner MO. Therapeutic potentials of GH secretagogues in adults. In Growth Hormone Secretagogues, pp 263-278. Eds E Ghigo, M Boghen, FF Casanueva \& C Dieguez. Amsterdam: Elsevier, 1999.

11 Shimon I \& Melmed S. Potential applications of growth hormone secretagogues. In Growth Hormone Secretagogues. pp 315-320. Eds E Ghigo, M Boghen, FF Casanueva \& C Dieguez. Amsterdam: Elsevier, 1999.

Received 12 February 2000

Accepted 15 February 2000 